\title{
Patterned Hydrogel Substrates for Cell Culture with Electrohydrodynamic Jet Printing
}

\author{
Michael J. Poellmann, Kira L. Barton, Sandipan Mishra, \\ Amy J. Wagoner Johnson*
}

Cells respond to and are directed by physiochemical cues in their microenvironment, including geometry and substrate stiffness. The development of substrates for cell culture with precisely controlled physiochemical characteristics has the potential to advance the understanding of cell biology considerably. In this communication, E-jet printing is introduced as a method for creating high-resolution protein patterns on substrates with controlled elasticity. It is the first application of E-jet printing on a soft surface. Protein spots as small as $5 \mu \mathrm{m}$ in diameter on polyacrylamide are demonstrated. The patterned hydrogels are shown to support cell attachment and spreading. Polyacrylamide substrates patterned by E-jet printing may be applied to further the study of cellular mechanobiology.

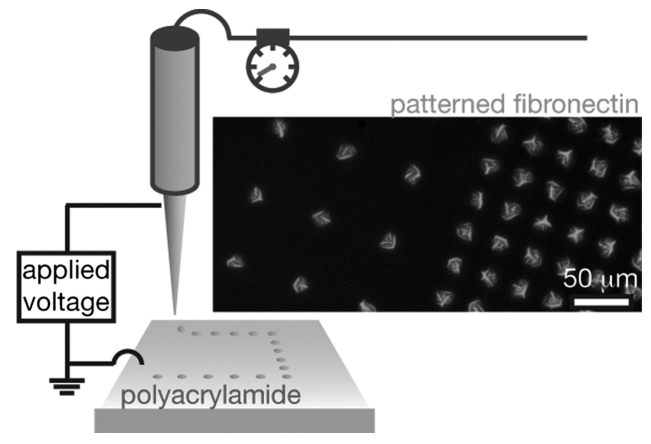

\section{Introduction}

The use of novel biomaterial systems for cell culture reveals how physiochemical factors in the extracellular environment direct cell behavior. In vitro studies with soft materials have shown that substrate elasticity influences cell shape, spreading, migration, proliferation, differentiation and extracellular matrix (ECM) production. ${ }^{[1-3]}$ Abnormally stiff microenvironments have been tied to pathophysiological conditions like the formation of fibrotic scar

Prof. A. J. Wagoner Johnson

Department of Mechanical Science and Engineering, University of Illinois at Urbana-Champaign, 1206 W. Green St., Urbana, IL 61801, USA

E-mail: ajwj@illinois.edu

Prof. K. L. Barton

Department of Mechanical Engineering, University of Michigan, 2350 Hayward St., Ann Arbor, MI 48109, USA

Prof. S. Mishra

Department of Mechanical, Aerospace, and Nuclear Engineering, Rensselaer Polytechnic Institute, $1108^{\text {th }}$ St., Troy, NY 12180, USA M. J. Poellmann

Department of Bioengineering, University of Illinois at UrbanaChampaign, 1304 W. Springfield Ave., Urbana, IL 61801, USA tissue and the malignant phenotype of cancer cells. ${ }^{[2]}$ Separate experiments demonstrate that cells are also highly sensitive to the type and spatial arrangement of ECM proteins. ${ }^{[4]}$ The micro-scale geometry of adhesive proteins influences cell structure and shape, migration, proliferation, and gene expression. ${ }^{[4,5]}$ Larger patterns tens of micrometers in size influence cellular survival and differentiation, while the differentiation and proliferation of individual cells in patterned aggregates (hundreds of micrometers in size) depend on geometric constraints determined by their location within the larger aggregate. ${ }^{[5]}$ The development of biomaterial systems with well-defined and independently-controlled physiochemical cues is critical to understanding how cues like substrate elasticity and ECM geometry interact to influence cell behavior. ${ }^{[6]}$

Polyacrylamide is a particularly useful biomaterial for in vitro studies, and is widely used to determine the influence of substrate elasticity on cell behavior. ${ }^{[7]}$ Polyacrylamide hydrogels have well-defined mechanical properties that can be tuned to match the elasticity of the softest tissues in the body ${ }^{[8]}$ Additionally, the gels resist protein adsorption in their unmodified state so that cells only attach and spread on areas that are specifically modified with adhesive ligands. ${ }^{[7]}$ 
In this paper, we introduce electrohydrodynamic jet (Ejet) printing ${ }^{[9,10]}$ as a new method for printing ECM proteins with micro-scale pattern features on polyacrylamide substrates. In E-jet printing, an ink solution is placed in a sealed reservoir with a conductive nozzle. With the right balance between capillary force and the applied backpressure on the fluid, the ink forms a spherical meniscus at the tip of the nozzle. Applying a potential difference between the nozzle and the substrate creates an electric field, pulling the fluid meniscus into a cone. It is through jetting from the tip of this cone that E-jet printing achieves high resolution. ${ }^{[9]}$ Droplet size and jetting frequency depend on the back-pressure, the separation distance between nozzle and substrate, and the applied voltage. The system can be operated in either direct current (DC) mode with droplets at a constant frequency or jetting in a continuous stream, or in pulsed-voltage mode with droplets controlled by a user-defined square wave voltage signal. ${ }^{[11]}$

Most notably, this paper represents the first application of E-jet on a soft substrate. Protein printing is demonstrated on polyacrylamide substrates with different elasticities. We also demonstrate cell attachment and spreading on printed fibronectin. The feature sizes obtained are significantly smaller than single cells, and are comparable to or smaller than other methods for patterning hydrogel cell substrates, including inkjet printing ${ }^{[12,13]}$ and microcontact printing. ${ }^{[5]}$ E-jet printing is a promising technique for creating substrates with subcellular-scale protein patterns on surfaces with well-defined mechanical properties for use in mechanobiology studies.

\section{Experimental Section}

\section{Substrate Preparation}

Glass cover slips were cleaned in a dilute soap solution then rinsed several times with de-ionized (DI) water and once with ethanol. The glass was prepared for gel attachment by treating for 10 min under 2 vol\% 3-(trimethoxysilyl)propyl methacrylate (Sigma) in 95 vol\% ethanol, pH 5, and placed on a $60^{\circ} \mathrm{C}$ hotplate for $10 \mathrm{~min}$. A prepolymer solution consisting of 8 vol\% acrylamide (Bio-Rad), 0.2 vol\% $N, N^{\prime}$-methylenebis(acrylamide) (bis) (Bio-Rad), and 0.2 vol\% acrylic acid (Sigma) was prepared in $0.137 \mathrm{M} \mathrm{NaCl}$ and $0.050 \mathrm{M}$ 4-(2-hydroxyethyl)-1-piperazineethanesulfonic acid (HEPES) (Sigma), pH 8. Polymerization was initiated by adding $1 /$ $20010 \mathrm{wt} \%$ ammonium persulfate (Bio-Rad) and 1/1000 N,N,N, $N^{\prime}$ tetramethylethylenediamine (Bio-Rad). For softer (ca. $9 \mathrm{kPa}$ ) and stiffer (ca. $35 \mathrm{kPa}$ ) gels, $6.0 \% / 0.15 \%$ and $10.0 \% / 0.25 \%$ acrylamide/bis were used, respectively. Droplets of prepolymer were sandwiched between an activated cover glass and a flat polystyrene surface. Gels were allowed $30 \mathrm{~min}$ to polymerize, then were washed overnight in DI water with 20 vol\% glycerin (Fisher). To activate, the gels were treated for $10 \mathrm{~min}$ under droplets of $0.015 \mathrm{~m}$ 1-ethyl-3-(3dimethylaminopropyl) carbodiimide hydrochloride (EDC) (Pierce) and $0.025 \mathrm{M} \mathrm{N}$-hydroxysuccinimide (NHS) (Pierce) in $0.050 \mathrm{M} \mathrm{2-(N-}$ morpholino)ethane sulfonic acid (Sigma), rinsed with DI water, placed on a $60^{\circ} \mathrm{C}$ hotplate for $5 \mathrm{~min}$. Activated gels were stored in a dry environment prior to printing.

\section{E-Jet Printing}

The glass-bonded and activated hydrogels were placed on a 5-axis computer-controlled stage and connected to ground. IgG ink solutions consisted of $5 \mathrm{mg} \cdot \mathrm{mL}^{-1}$ rabbit Immunoglobulin-G (Pierce), 20 vol\% glycerin, and 0.1 vol\% Tween-20 (Sigma) in phosphate buffered saline (PBS) (Lonza). Fibronectin inks contained $0.5 \mathrm{mg} \cdot \mathrm{mL}^{-1}$ human fibronectin (BD Biosciences) in salt-free 0.050 M HEPES, pH 8 with $0.1 \%$ Tween-20 with 20 or 40 vol\% glycerin for printing with $5 \mu \mathrm{m}$ and $2 \mu \mathrm{m}$ inner diameter nozzles, respectively. Ink was loaded in a pressure-sealed syringe clamped above the substrate with Luer-tipped micropipette nozzles (World Precision Instruments). Prior to printing, the nozzles were sputter-coated with a $5 \mathrm{~nm}$ layer of $\mathrm{Au} / \mathrm{Pd}$ for conductivity and treated for $3 \mathrm{~min}$ in $1 \mathrm{H}, 1 \mathrm{H}, 2 \mathrm{H}, 2 \mathrm{H}^{-}$ perfluorodecane-1-thiol (Sigma). Voltage, substrate alignment, nozzle separation distance, and stage displacement were controlled through a custom-developed LabVIEW interface. Printing was monitored with an Infinity 3 camera (Luminera Scientific) with an Edmund Industrial Optics lens. Following printing, substrates were stored in a sealed container at room temperature for at least $4 \mathrm{~h}$, then thoroughly rinsed in PBS to rehydrate and remove unreacted NHS groups. For cell culture, gels were sterilized by briefly soaking in $70 \mathrm{vol} \%$ ethanol then transferred to sterile $6 \mathrm{~cm}$ plates and rinsed several times with cell media.

\section{Pattern Characterization}

Hydrogels were rehydrated in phosphate buffered saline. IgG proteins were detected with DyLight 488-conjugated goat antirabbit secondary antibodies (Pierce) following treatment with a solution of 1 vol\% goat serum (Pierce). Fibronectin was visualized after treatment with sheep anti-human fibronectin IgG (R\&D Systems) and NL493-conjugated donkey anti-sheep IgG (R\&D Systems). Images were obtained with a OImaging Retiga 2000R camera mounted on a Leica DMI 4000 B inverted fluorescent microscope. A Zeiss 710 confocal microscope was used for 3D imaging a $3 \times 10$ array of $10 \mu \mathrm{m}$ diameter spots and a $2 \times 6$ array of $20 \mu \mathrm{m}$ spots. Depth of penetration was determined using Zeiss ZEN software.

\section{Cell Culture}

Hydrogels patterned with fibronectin were sterilized in $70 \%$ ethanol and rinsed several times with PBS and media prior to cell seeding. D1 ORL UVA mouse mesenchymal stem cells (ATCC) and primary rat mesenchymal stem cells (Millipore) were grown at $37^{\circ} \mathrm{C}$ in a humidified incubator under Dulbecco's Modified Eagle Medium-low glucose, modified to contain $4 \times 10^{-3} \mathrm{M} \mathrm{L}$-glutamine, $1 \times 10^{-3} \mathrm{M}$ sodium pyruvate, and $3.7 \mathrm{~g} \cdot \mathrm{L}^{-1}$ sodium bicarbonate (UIUC Cell Media Facility) with $10 \%$ added fetal bovine serum (Gibco) and $100 \mathrm{U} \cdot \mathrm{mL}^{-1}$ penicillin-streptomycin (Mediatech). The D1 cells were lifted at passage 10-12 with $0.05 \%$ trypsin-EDTA (Invitrogen), seeded on substrates at a density of $10^{5}$ cells per dish, and observed with phase contrast microscopy after $3 \mathrm{~d}$. Primary cells were lifted at passage 3 , seeded at a density of $5 \times 10^{4}$ cells per

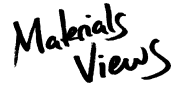

www.MaterialsViews.com 
dish. After $24 \mathrm{~h}$, the cells were fixed with neutral buffered formalin (Sigma), treated with 1 vol\% Triton X-100 (Sigma), and stained with rhodamine phalloidin (Invitrogen) following the fibronectin antibody treatment. Image brightness, contrast, and coloring was adjusted using NIH ImageJ software was used to make composite fluorescent images.

\section{Results and Discussion}

The polyacrylamide substrates contain an added $0.2 \%$ acrylic acid into the polymer backbone. Those groups are subsequently modified with EDC/NHS to activate the gel for protein attachment. When proteins contact the activated hydrogel, amine-containing amino acids displace the NHS groups and form covalent bonds directly to the polymer backbone. ${ }^{[14]}$ Fully-hydrated substrates shrink during printing, which is undesirable because it is critical to maintain a consistent distance between the printer nozzle and the substrate. To control for this, we baked the substrates on a hotplate to remove most of the water prior to printing. Aside from maintaining a constant stand-off height, a dry environment also better protects the activity of NHS groups. ${ }^{[14]}$ Following printing, the remaining NHS groups are hydrolytically cleaved when the substrates are rehydrated with PBS, making unpatterned regions resistant to further protein adsorption. The gel activation and protein conjugation are summarized in Figure 1.

The activated substrates are electrically grounded and placed on a vacuum chuck attached to a computercontrolled translating stage for printing. The protein ink (described below) is loaded into a reservoir, below which there is a glass capillary nozzle that is sputter-coated with gold-palladium for enhancing conductivity. Ink droplet size and jetting frequency are controlled by computer-defined voltage and back-pressure. The E-jet system is illustrated in Figure 2.

We first printed rabbit immunoglobulin G (IgG) as a model protein in DC mode with the ink solutions containing $5 \mathrm{mg} \cdot \mathrm{mL}^{-1} \mathrm{IgG}, 20 \%$ glycerin, and $0.05 \%$ Tween-20. Glycerin increases the viscosity of the solution and mitigates evaporation from the meniscus, which can cause the nozzle to clog. Tween-20 helps prevent protein aggregation. Printing typically occurs with a back-pressure of 1-3 psi and an applied voltage of 250-350 V. Nozzles have $5 \mu \mathrm{m}$ inner diameter and are kept at a consistent stand-off height of $30 \mu \mathrm{m}$ above the substrate. Droplets of various sizes are demonstrated on hydrogels with elastic moduli

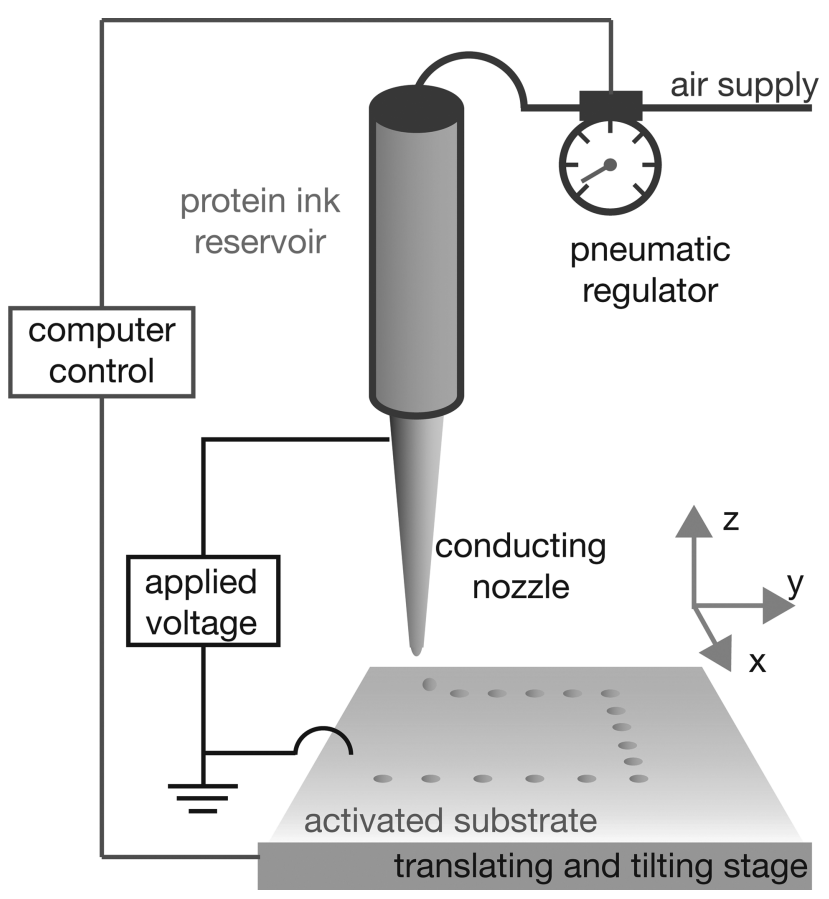

Figure 2. Diagram of E-jet setup. The balance between the applied voltage, back pressure applied to the ink reservoir, and capillary pressure in the nozzle determines droplet size and frequency. The activated polyacrylamide hydrogel is grounded and attached to a translating and tilting computer-controlled stage. 
ranging from 9.6 to $34.4 \mathrm{kPa}$, a range that is known to influence MSC differentiation. ${ }^{[8]}$ Following printing, IgGpatterned gels are washed and treated with fluorescent antibodies for visualization (Figure 3a-c). Confocal microscopy shows that proteins are located at the surface of gels where they would be accessible to cells (Figure 3c), but also penetrate approximately $30 \mu \mathrm{m}$ into the gel.

ECM protein inks consisted of $0.5 \mathrm{mg} \cdot \mathrm{mL}^{-1}$ fibronectin and $0.05 \%$ Tween-20. To obtain features with higher resolution, we use $2 \mu \mathrm{m}$ nozzles. We increase the glycerin content to $40 \%$ to reduce the potential for clogging the smaller nozzle. Substrates were treated with a primary antibody that binds fibronectin and a secondary antibody conjugated to a fluorophore (green) (Figure 3d-f). Spots and lines smaller than $5 \mu \mathrm{m}$ across were obtained, with the smallest spots obtained using pulse control (Figure 3e). Substrates were seeded with mesenchymal stem cells following printing. The cells were allowed to attach and spread overnight before fixing and imaging. Primary mesenchymal stem cells were stained with rhodamine phalloidin, which binds to filamentous actin and appears red (Figure 4a-c). Additionally, D1 cells were patterned and imaged using phase contrast microscopy after 3 days of culture (Figure $4 \mathrm{~d}, \mathrm{e}$ ). Cells were observed to adhere only to regions where fibronectin had been printed.

E-jet printing possesses superior qualities when compared with conventional inkjet printing, namely, a higher feature resolution. E-jet printed features are an order of magnitude smaller than inkjet printing, enabling patterning of single cells. Only microcontact printing offers comparable resolution, achieving line widths of less than $10 \mu \mathrm{m} \cdot{ }^{[15-17]}$ However, the main disadvantage of microcontact printing is its inflexibility. Once fabricated, stamps cannot be altered, and fabricating a new stamp requires a clean room facility and a relatively high level of expertise. E-jet printing, in comparison, has high process flexibility and the pattern can be controlled at the point of printing. Additionally, E-jet systems can be assembled at relatively low cost. ${ }^{[18]}$
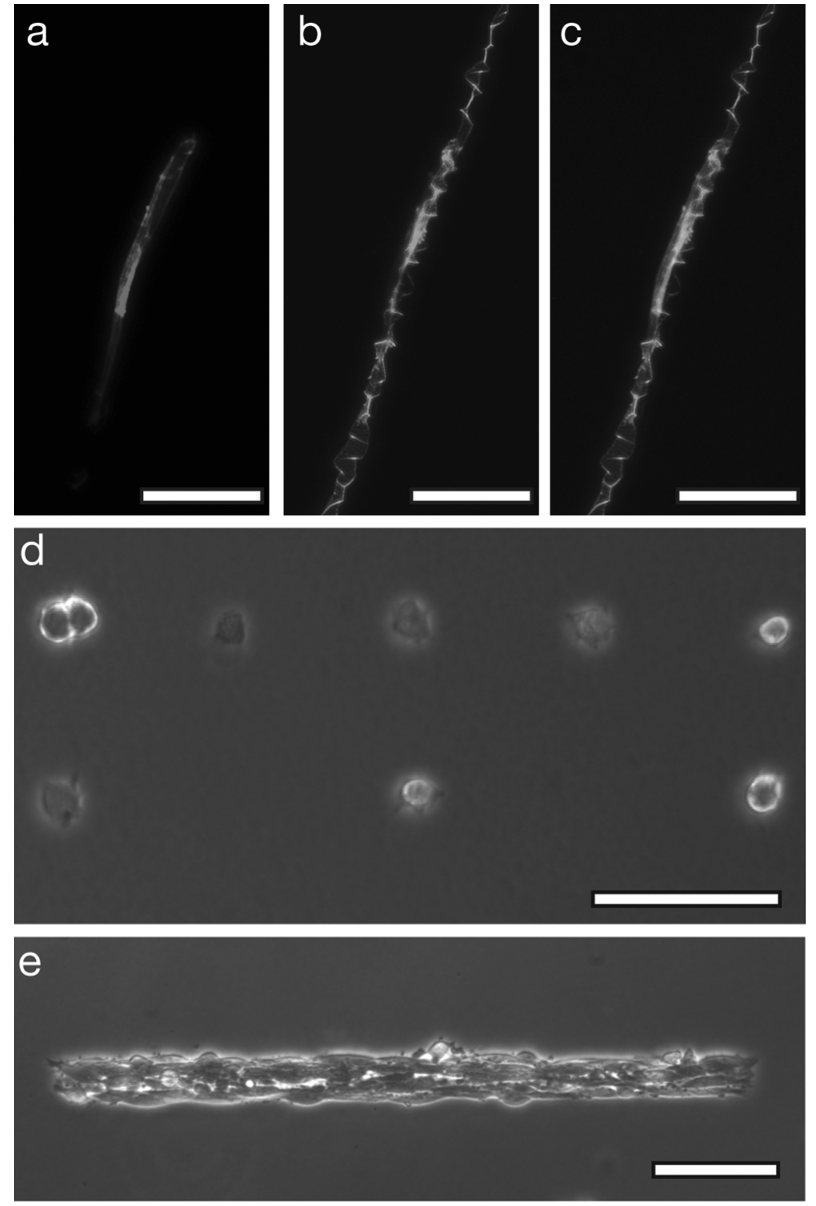

Figure 4. A primary mesenchymal stem cell stained for filamentous actin (a) elongated and aligned with a fibronectin stripe (b) detected with immunofluorescence. A composite image is shown in (c). A phase contrast image of D1 cells adhered to equally spaced spots of fibronectin (d). Spots can be printed with a small enough diameter to constrain single cells. A phase contrast image of densely packed D1s on a narrow rectangle of patterned fibronectin (e). Scale bars are $50 \mu \mathrm{m}$.
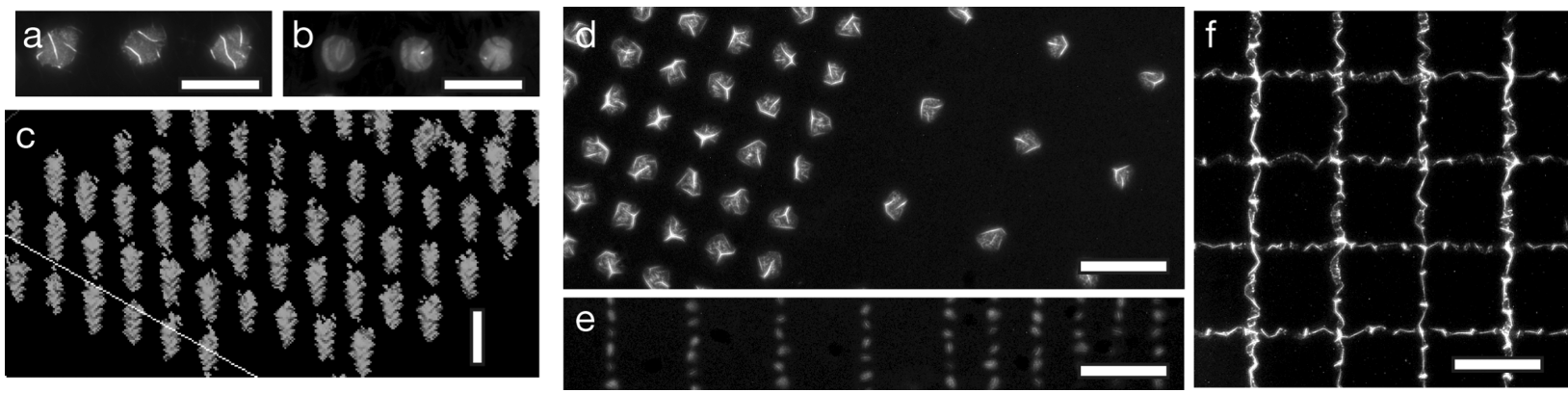

Figure 3. Droplets of IgG printed on $9.6 \mathrm{kPa}$ (a) and $34.4 \mathrm{kPa}$ (b) gels. Scale bars are $50 \mu \mathrm{m}$. Confocal image shows that IgG was located at the surface, but penetrated approximately $30 \mu \mathrm{m}$ into a $21.1 \mathrm{kPa}$ gel (c). Immunofluorescent detection of printed fibronectin on $21.1 \mathrm{kPa}$ gels showing precise placement with spot sizes down to approximately $5 \mu \mathrm{m}$ in diameter (d-f). Scale bars are $50 \mu \mathrm{m}$.

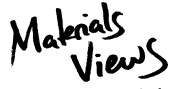

www.MaterialsViews.com
Macromol. Biosci. 2011, 11, 1164-1168

(C) 2011 WILEY-VCH Verlag GmbH \& Co. KGaA, Weinheim 


\section{Conclusion}

We report a technique for patterning cell adhesion proteins with high resolution on polyacrylamide hydrogels using Ejet printing. The use of a hydrogel substrate is a novel application for E-jet, which is capable of creating patterns with dimensions significantly smaller than a single cell. This method can be employed to create patterned ECM proteins on surfaces with precisely controlled physical properties for cell culture.

Acknowledgements: This material is based on work supported by the Center for Nanoscale Chemical Electrical Mechanical Manufacturing Systems at the University of Illinois, funded by the National Science Foundation under Grant DMI-0328162. We would like to acknowledge Hannah Friedman and Chelsea Frid for their help with hydrogel fabrication, and Shiv Sivaguru at the Institute for Genomic Biology at the University of Illinois for the confocal image.

Received: January 6, 2011; Published online: June 7, 2011; DOI: 10.1002/mabi.201100004

Keywords: electrohydrodynamic jet; polyacrylamide; hydrogels; proteins; surface modification

[1] S. R. Peyton, C. M. Ghajar, C. B. Khatiwala, A. J. Putnam, Cell Biochem. Biophys. 2007, 47, 2.
[2] P. A. Janmey, J. P. Winer, M. E. Murray, O. Wen, Cell Motil. Cytoskeleton 2009, 66, 8.

[3] D. E. Discher, P. Janmey, Y. Wang, Science 2005, 310, 5751.

[4] B. Geiger, J. P. Spatz, A. D. Bershadsky, Nat. Rev. Mol. Cell Biol. 2009, 10, 1.

[5] W. F. Liu, C. S. Chen, Adv. Drug Delivery Rev. 2007, 59, 13.

[6] J. Y. Wong, J. B. Leach, X. O. Brown, Surf. Sci. 2004, 570, 1.

[7] C. E. Kandow, P. C. Georges, P. A. Janmey, K. A. Beningo, in: Cell Mechanics, Vol. 83 Y. L. Wang, D. E. Discher, Eds., Academic Press, Missouri, USA 2007.

[8] A. J. Engler, S. Sen, H. L. Sweeney, D. E. Discher, Cell 2006, $126,4$.

[9] J. Park, M. Hardy, S. J. Kang, K. Barton, K. Adair, D. Mukhopadhyay, C. Y. Lee, M. S. Strano, A. G. Alleyne, J. G. Georgiadis, P. M. Ferreira, J. A. Rogers, Nat. Mater. 2007, 6, 10.

[10] J. Park, J. H. Lee, U. Paik, Y. Lu, J. A. Rogers, Nano Lett. 2008, 8, 12.

[11] S. Mishra, K. L. Barton, A. G. Alleyne, P. M. Ferreira, J. A. Rogers, J. Micromech. Microeng. 2010, 20, 9.

[12] S. Ilkhanizadeh, A. I. Teixeira, O. Hermanson, Biomaterials 2007, 28, 27.

[13] E. Roth, T. Xu, M. Das, C. Gregory, J. Hickman, T. Boland, Biomaterials 2004, 25, 17.

[14] G. T. Hermanson, "Bioconjugate Techniques", $2^{\text {nd }}$ edition Academic Press, Missouri, USA 2008.

[15] M. Burnham, J. Turner, D. Szarowski, D. Martin, Biomaterials 2006, 27, 35.

[16] M. R. Hynd, J. P. Frampton, M. R. Burnham, D. L. Martin, N. M. Dowell-Mesfin, J. N. Turner, W. Shain, J. Biomed. Mater. Res. A 2007, 81, 2.

[17] V. Damljanović, B. C. Lagerholm, K. Jacobson, BioTechniques 2005, 39, 6 .

[18] K. Barton, S. Mishra, K. Shorter, A. Alleyne, P. Ferreira, J. Rogers, Mechatronics 2010, 20, 5. 